\title{
Development of innovative indoor/outdoor air quality monitoring for environmental impact assessment in the State of Qatar
}

\author{
A. Alassi, A. Khan, D. Ayesh, I. Aljayyousi, R. Aldisi, S. Elasad, \\ F. Touati \& M. Benammar \\ Department of Electrical Engineering at Qatar University, Qatar
}

\begin{abstract}
Air pollution is one of the most crucial factors affecting the quality of life and health of increasingly urban populations; a country with a fast-growing economy based on hydrocarbons such as Qatar is no exception. In addition to the adverse impact of air pollution on humans, animals and plants, the presence of dust and air-borne particulate matter affect negatively the efficiency of solar panels used for power generation. In this project, a comprehensive wireless solution is proposed for monitoring and measuring the levels of some known harmful gases such as $\mathrm{CO}_{2}, \mathrm{NO}_{2}$ and $\mathrm{CH}_{4}$ as well as other environmental parameters such as dust, temperature, humidity, solar irradiance, pressure, wind-direction and wind speed. Data is collected by appropriate sensor nodes (NI-WSN) that communicate wirelessly (using IEEE 802.15.4 protocol) with a host computer (Gateway) that receives, processes, stores and displays the collected information via LabVIEW environment, which represents the software part of the project (Programming and GUI Design). The data processing part includes analysing the environmental parameter levels, which is later used to study the efficiency of using solar panels in Qatar. The complete system has been implemented and successfully tested in a chosen site within Qatar University Campus. In the long term, we hope that the data collected from our air quality monitoring system will help in areas of research related to environmental monitoring and developing systems that correlate indoor and outdoor readings to the operation of an indoor HVAC system.
\end{abstract}

Keywords: air quality monitoring, air pollution, intelligent sensors, computer aided data analysis, environmental engineering and management. 


\section{Introduction}

Wireless Sensor Networks (WSNs) consist of spatially-distributed autonomous sensors connected via wireless communication links. The first wireless sensor networks had military applications such as the DARPA Distributed Sensor Nets (DSN, CMU) in 1978, DARPA SensIT in 1998, and NASA Sensor Webs Project in 2001. Wireless Integrated Network Sensors (WINS) technology - that began to appear with the commercialization of the Internet - has made it possible to access the wireless sensor network through an Internet network. Such projects were carried out in UCLA University in 1993, and the Stardust project developed in UC Berkeley in 1998 [1].

Air pollution is one the most crucial factors affecting the quality of life and the health of increasingly urban population of industrial states such as the State of Qatar [2-4]. The deployment of wireless sensor networks allows for continuous monitoring of fresh air condition in both indoor and outdoor areas.

The issue of air quality conditions and pollution in the Gulf Cooperation Council (GCC) countries took the attention due the high production and consumption of hydrocarbon energy in the region $[5,6]$. There are increased percentages of harmful gases emissions in the atmosphere. The GCC countries constitute $0.6 \%$ of the world population; however they contribute to $2.4 \%$ of the global greenhouse gas emissions [7]. Such percentages are powered, in addition to the industry, by the huge number of cars in the roads. The low prices of fuel in the regions compared to Europe and the U.S. is supporting the growth in usage, where you can buy a liter of petrol for less than one third of a dollar, which is even cheaper than one liter of soft drinks!

One of the neglected sources of pollutant in GCC countries, beside the Greenhouse gases emissions, is dust. Dust and sand create serious health problems to people with asthma and respiratory system related diseases. Moreover, the dust negatively affects the solar panels efficiency, and researches are conducted in this matter [8].

Furthermore, there are pollutants that are generated by the industrial areas. For example, in Ras Laffan City, there are many companies for LNG production, which can generate lethal pollutants such as Hydrogen Sulfide. During the LNG production, with hydrocarbon flow from offshore to onshore; some toxic gases accompany the natural gas such as Hydrogen Sulfide $\mathrm{H}_{2} \mathrm{~S}$. Such pollutants have adverse effects on health, and that is why institutes such as the World Health Organization (WHO) impose restrictions on the pollutant levels [9].

In Qatar, the main sources of pollution are dust particulates, Greenhouse gases and toxic gases emissions generated by industry. Therefore, the monitoring system should include these three sources in addition other important parameters such as temperature, pressure and humidity since it is known that they have correlation with the remaining parameters. 


\section{System objectives and methodology}

The air quality monitoring system aims to record and analyse different parameters that affect the weather both indoor and outdoor. The parameters to measure are:

- Ambient temperature.

- Atmospheric pressure.

- Humidity.

- Solar irradiance.

- Dust.

- Carbon Dioxide $\left(\mathrm{CO}_{2}\right)$.

- Carbon Monoxide (CO).

- Nitrogen Dioxide $\left(\mathrm{NO}_{2}\right)$.

- Sulfur Dioxide $\left(\mathrm{SO}_{2}\right)$.

- Methane $\left(\mathrm{CH}_{4}\right)$.

The system will be designed to collect data using WSN nodes located at two different locations, indoors and outdoors, and sent to the central unit. The number of sites can be easily extended to include any number of sites. The measurements taken will differ according to the location. Both indoors and outdoors location are in college of engineering building in Qatar University. The following measurements will be taken indoors in a lab:

- Ambient temperature.

- Atmospheric pressure.

- Humidity.

- Carbon Dioxide $\left(\mathrm{CO}_{2}\right)$.

- Carbon Monoxide (CO).

- Nitrogen Dioxide $\left(\mathrm{NO}_{2}\right)$.

- Sulfur Dioxide $\left(\mathrm{SO}_{2}\right)$.

- Methane $\left(\mathrm{CH}_{4}\right)$.

And the following measurements will be taken outdoors:

- Ambient temperature.

- Atmospheric pressure.

- Humidity.

- Solar irradiance.

- Dust.

- Carbon Dioxide $\left(\mathrm{CO}_{2}\right)$.

- Carbon Monoxide (CO).

- Nitrogen Dioxide $\left(\mathrm{NO}_{2}\right)$.

- Sulfur Dioxide $\left(\mathrm{SO}_{2}\right)$.

- Methane $\left(\mathrm{CH}_{4}\right)$. 


\section{Network design}

The system aims to collect the different sensors measurements in three different locations ( 2 indoor and 1 outdoor). Nevertheless, increasing the number of locations can be done easily since the methodology followed in the network design to collect the sensors outputs such as using NI wireless nodes used in acquisition. Each NI node has a limited number of analog inputs, however, using with the usage of an analog multiplexer, the number of inputs increases which is a very economical solution that was used to decrease the number of nodes at each site to one node only. The outdoor parameters measured are slightly different than the outdoor parameters measured based on the need; in the indoors the solar irradiance and the dust are not measured.

One NI wireless node is place at each location for data acquisition with one analog multiplexer and the required conditioning circuits for the sensors.

\subsection{Sensors design and conditioning circuits}

\subsubsection{Temperature sensor}

LM35 sensor is used for measuring the temperature. This sensor gives an output voltage that is directly proportional to the temperature in Celsius of the surrounding environment.

The datasheet of LM35 says that the constant of proportionality between output voltage and temperature is $100 \mathrm{C}^{\mathrm{o}} / \mathrm{V}$. Specifically, multiplying the output voltage by the factor $100 \mathrm{C}^{\mathrm{o}} / \mathrm{V}$ to obtain the surrounding temperature.

\subsubsection{Humidity sensor}

HSM-20G sensor was used to measure the humidity; this sensor converts the humidity to a voltage signal. In addition, the conditioning circuit was illustrated in the sensor datasheet with the typical calibration data.

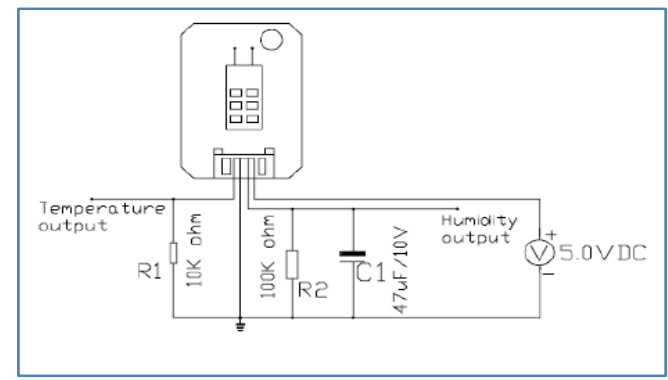

Figure 1: HSM-20G (humidity sensor) conditioning circuit.

The humidity can be obtained by this equation

$$
\text { Humidity }(\%)=\frac{\text { Vout }-0.3792}{0.0323}
$$




\subsubsection{Pressure sensor}

Measuring the pressure was done by MPX2050GP sensor; it is a piezoresistive sensor. The sensor gives a differential voltage that is directly proportional to the differential pressure applied. The figure below shows the structure of the sensor The pressure is obtained by this equation

$$
P(k P a)=\frac{\text { Vo(differential })+0.2359}{0.1223}
$$

\subsubsection{Solar irradiance sensor}

The self-powered Pyranometer SP-110 used in measure the solar irradiance; the sensor gives a differential output ranges from $(0-250 \mathrm{mV})$. However, a conditioning circuit attached to this sensor to change its range to $(0-5) \mathrm{V}$, this is done by INA121 instrumentation amplifier with gain of 20. Eventually, the equation is

$$
\text { Total Radiation }\left(W m^{2}\right)=\left(\frac{\text { Sensor Differential Output }(V)}{4}\right)
$$

\subsubsection{Dust sensor}

This project uses GP2Y1010AU0F dust sensor. This sensor operation is based on the pulse signal applied to LED terminal in order to activate the LED inside the sensor. The light emitted from the LED is reflects on the photodiode. The output level measured from the photodiode amplifier indicates the concentration of dust. Figure 2 shows the conditioning circuit.

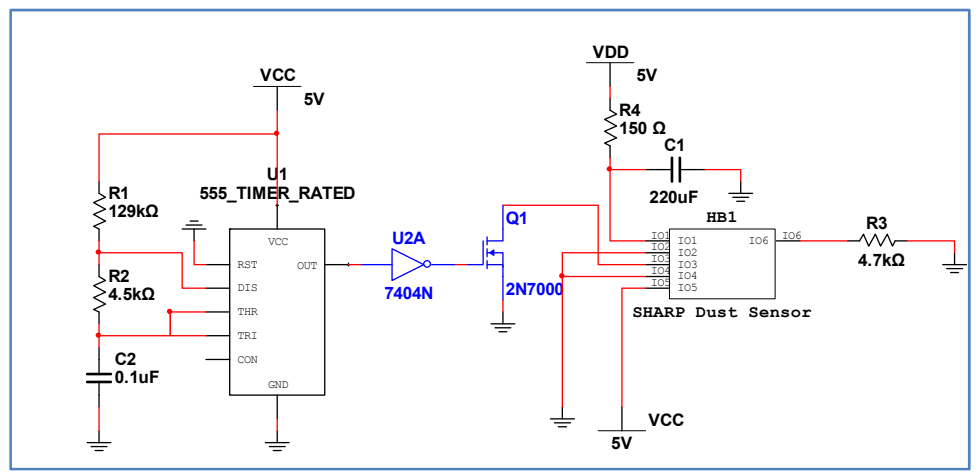

Figure 2: GP2Y1010AU0F (dust sensor) circuit.

The relation between the sensor's output and the dust density follows this equation

$$
\text { Dust Density }\left(\mathrm{mg} / \mathrm{m}^{3}\right)=\frac{V_{\text {out }}-0.5456}{5.8213}
$$




\subsubsection{Carbon Dioxide $\left(\mathrm{CO}_{2}\right)$ sensor}

MG811 sensor was chose to measure the concentration of $\mathrm{CO}_{2}$ gas; since it has good sensitivity and low dependency on temperature and humidity. The sensor has an acceptable range of detection (350-10,000) ppm. Figure 3 shows the circuit diagram of MG811.

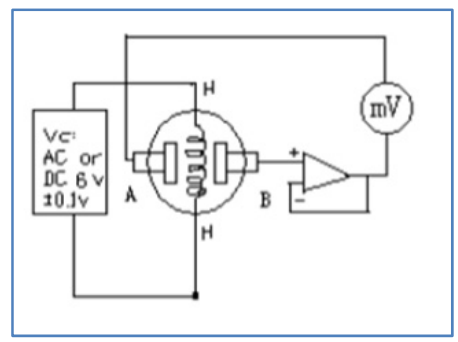

Figure 3: $\quad \mathrm{MG811}\left(\mathrm{CO}_{2}\right.$ sensor $)$ circuit.

The sensor gives a voltage output between (30-50) $\mathrm{mV}$. Getting a suitable output voltage, an amplifier with a gain of 100 is used. The datasheet provides a relationship between the sensor's output voltage and the concentration of $\mathrm{CO}_{2}$.

\subsubsection{Nitrogen Dioxide $\left(\mathrm{NO}_{2}\right)$ sensor}

$\mathrm{NO}_{2}$ concentration is measured with MiCS-2710 sensor. The sensor detection range is $(0.05-5) \mathrm{ppm}$, and figure 4 shows the sensor conditioning circuit.

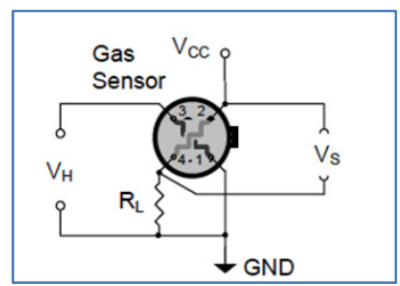

Figure 4: $\mathrm{NO}_{2}$ sensor circuit.

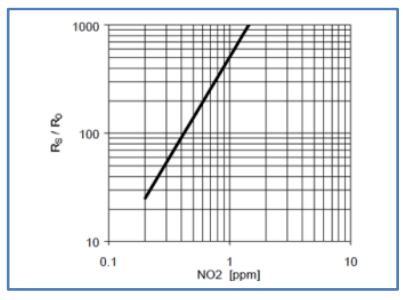

Figure 5: Sensor's resistance vs. concentration.

where $\mathrm{V}_{\mathrm{H}}$ is $(1.7 \mathrm{~V}), \mathrm{V}_{\mathrm{CC}}$ is $(2.5 \mathrm{~V}), \mathrm{R}_{\mathrm{L}}$ is $(10 \mathrm{k} \Omega)$ and $\mathrm{V}_{\mathrm{S}}$ is the output voltage. The sensor's datasheet provides a relationship between the sensor's internal resistance and gas concentration (shown in figure 5). The resistance is found using the following equation

$$
R_{s}=\frac{R_{L}}{\left(V_{c c}-V_{S}\right)} V_{S}
$$




\subsubsection{Sulphur Dioxide $\left(\mathrm{SO}_{2}\right)$ sensor}

MQ136 is used to measure the concentration of $\mathrm{SO}_{2}$, which has a simple circuit shown in figure 6 . The sensor can detect concentrations of SO2 between (1-200) ppm.

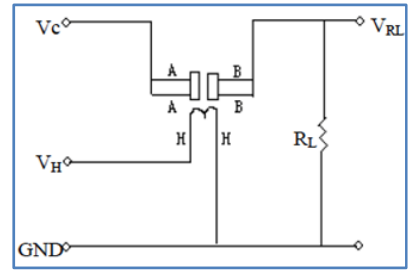

Figure 6: $\mathrm{MQ136}\left(\mathrm{SO}_{2}\right.$ sensor $)$ circuit.

where $\mathrm{V}_{\mathrm{H}}$ and $\mathrm{V}_{\mathrm{C}}$ are both $5 \mathrm{~V}, \mathrm{R}_{\mathrm{L}}(2 \mathrm{k} \Omega)$, and $\mathrm{V}_{\mathrm{RL}}$ is the output voltage. The datasheet provides a relationship between the sensor's resistance and the output voltage.

\subsubsection{Methane $\left(\mathrm{CH}_{4}\right)$ sensor}

MQ4 sensor is used to measure the concentration of Methane, Propane and Butane, but we are only interested in measuring Methane. It can measure $\mathrm{CH}_{4}$ with concentration between $(300-10,000) \mathrm{ppm}$. The circuit applied for measurement is exactly the same as the $\mathrm{SO}_{2}$ measurement circuit shown in figure 6 .

\subsubsection{Carbon Monoxide (CO) sensor}

MQ7 gas sensor will be used to measure the concentration of $\mathrm{CO}$, which can detect concentrations between (20 and 2000) ppm. Figure 7 shows the measure circuit.

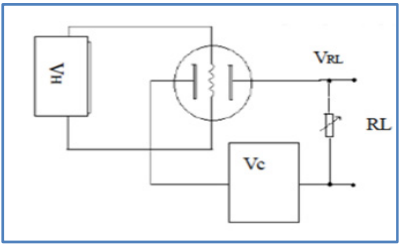

Figure 7: $\quad$ CO sensor circuit.

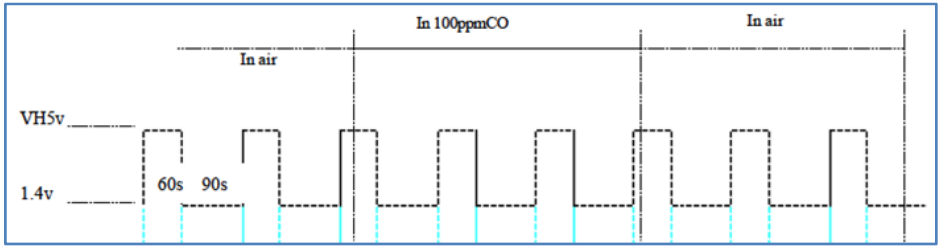

Figure 8: Heating voltage of MQ7 CO sensor. 
where $\mathrm{V}_{\mathrm{C}}$ is $(5 \mathrm{~V}), \mathrm{VH}$ is the heating voltage (shown in figure 8 ), $\mathrm{R}_{\mathrm{L}}$ is the load resistance, which was chosen to be $(2 \mathrm{k} \Omega)$ and $V_{R L}$ is the output voltage. MQ7 datasheet also provides a relationship between the gas concentration and the internal resistance of the sensor

\subsection{The powering circuit}

The indoor powering will be using a variable power supply device for all of the sensors. However, for the outdoor measurements, since no variable sources are available, a battery was used to supply the sensors' circuits. Furthermore, the voltages levels were selected based on the sensors need to provide accurate results. A 9 volts battery was used and is connected to a voltage regulator chip. The sensors are connected to the voltage regulator chip that gives an output of 5 volts. For the other sensors that need more than 5 volts due to op amps, they were connected directly to the battery.

\section{LabVIEW system design and features}

The signals sent by the WSN-3202 nodes is received by the NI-9792 Gateway, which is connected to a PC where National Instruments LabVIEW 2012 is used to process the data received from the nodes and to convert them into useful information. The first step is to read the data from the analog inputs; this step is done separately for each node, the input signals are then reverted back to produce values that represent their respective parameters. These results are then connected to numerical and graphical indicators, where three analog inputs are processed directly. The fourth input is the one connected to the analog multiplexer. This Mux used is an eight to one analog Multiplexer, so it has to be controlled using three digital switches, which are available in the WSN-3202 nodes. The controls have to be switched automatically in a continuous sequence to allow receiving data from different sensors. For this reason, for loop is used with a user-determined delay to switch between the different cases, and Case structures are used to set the output of each case.

The loop delay is controlled by the user, where he enters the desired delay, then this number is divided by the number of loop iterations and the result is multiplied by 1000 to get the loop delay in ms. This input delay is also considered as a reading period for the whole program, since the main code is running inside an infinite while loop that has to wait for the whole program to execute in order for it to go to the next iteration.

For the other two indoor nodes, the multiplexers read fewer inputs. However, the iterations and cases are maintained to 6 to keep the consistency of the whole system, and their loop delays are connected to the same delay as the outdoor loop through property nodes. These property nodes are used through the block diagram whenever connecting a variable from one of the internal case structures to the main program is required.

After obtaining the required inputs, some of the parameters - such as gas levels - have to be compared with their safety threshold values. Using a Boolean 


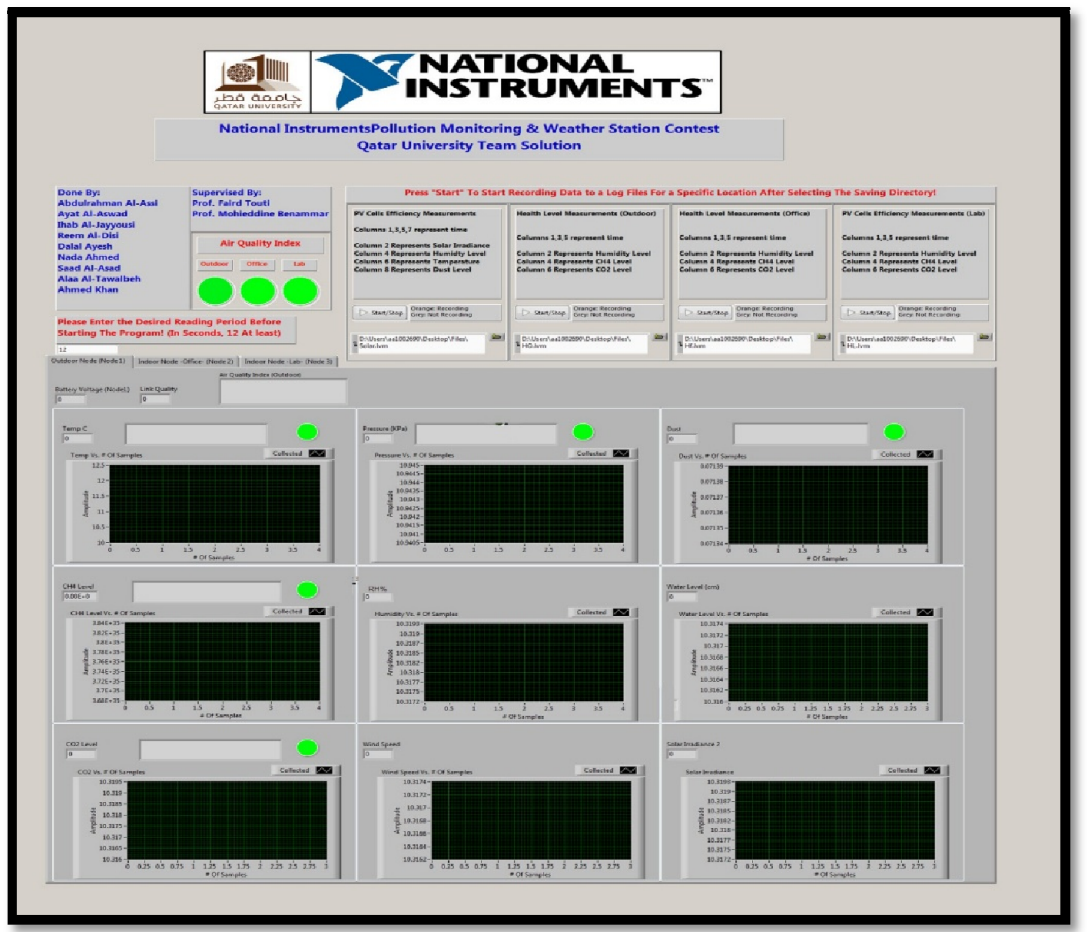

Figure 9: $\quad$ LabVIEW front panel.

comparator, where the result is compared with the threshold, does this and the output is then set as a control for another case structure that sets the output message depending on the comparator value. "The threshold was set to 0 in the figure above during the development stage and doesn't represent the real case".

In addition, an Air Quality Index was developed for each location to state if the weather is healthy depending on the comparators output. Property nodes were used to connect the comparators output from the different cases of the case structures. For the outdoor node, the AQ is considered good if temperature, pressure, gasses level and dust levels were below the safe threshold values. The same is true for the indoor nodes. The LabVIEW logic behind designing this idea is using an OR gate, if only one measured exceeds the threshold value, then the output of that gate will alter causing the output message to change.

Another feature that was added to the system was writing to measurement files. That is, the parameters related to the PV cells efficiency are stored in a log file, and the data for monitoring some environmental parameters that affect the health are stored in a different file for each location (Outdoor, Office and Lab). The user is given the choice of where to save the files and what to name them. Also, he is given the ability to enable or disable this process.

Moving to the LabVIEW front panel shown in figure 9, it was designed to be user friendly and easy to understand. The user has to enter the reading time as 
explained above. Also, the default case for writing to log files is set to be false, so the user is given the choice whether he wants to start recording from the beginning or later whenever he decides. The contents of the log files are also explained in terms of column headers.

For the Air Quality Index, the three circles at the top indicate the weather condition at the three different locations. The circle color change to red if something abnormal is detected, following the location. This generalized monitoring system allows the user to accurately detect the source of abnormality by choosing the tab corresponding to the affected location and noticing the levels of all the parameters.

Nodes battery voltage and Link Quality with the Gateway are also shown locally for each location on the front panel, so that the user can monitor them and act according to their level.

Figure 10 shows the flowchart of the whole LabVIEW block diagram.

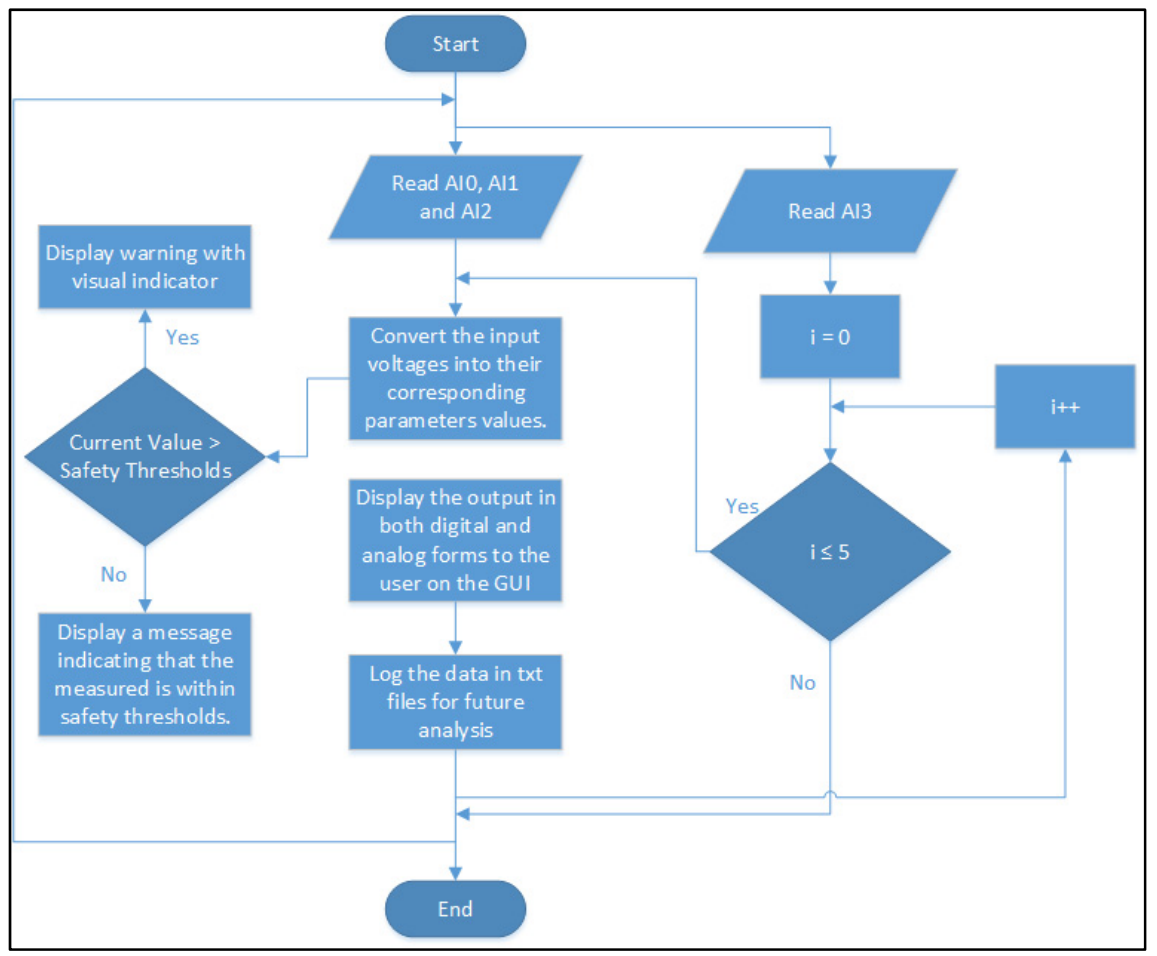

Figure 10: Flowchart of the whole LabVIEW block diagram.

\section{Results}

The system was connected and tested for both indoor and outdoor conditions. The used sensors were dust, humidity, temperature, pressure and solar irradiance. 
The solar irradiance was excluded for the indoor case as the light intensity is insignificant. Batteries were used to power the sensors and the analog multiplexer, as well as the digital output of the nodes.

The sensor readings were wirelessly transferred using the WSN-3202 nodes, received by the 9792 -Gateway and interpreted by LabVIEW, where the voltage levels were converted into their respective parameter values. Both indoor and outdoor systems were activated for a period of time, and the results were obtained separately for testing purposes, and they were displayed on the front panel. An example for the indoor case is shown below in figure 11.

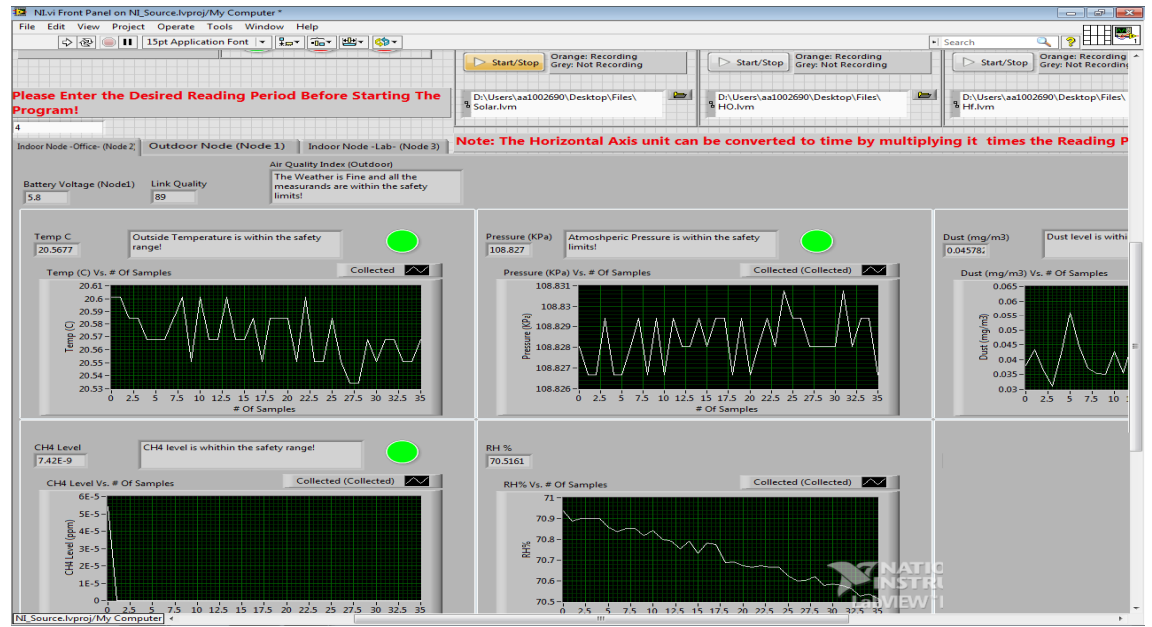

Figure 11: LabVIEW front panel results for indoor case.

To demonstrate the data recording validity: temperature, dust and humidity data were logged and used later to re-generate the graphs of these parameters. This feature is useful for long term monitoring where dividing the data into subperiods is required. An example of these graphs is shown in figure 12, where MS Excel was used for plotting them:

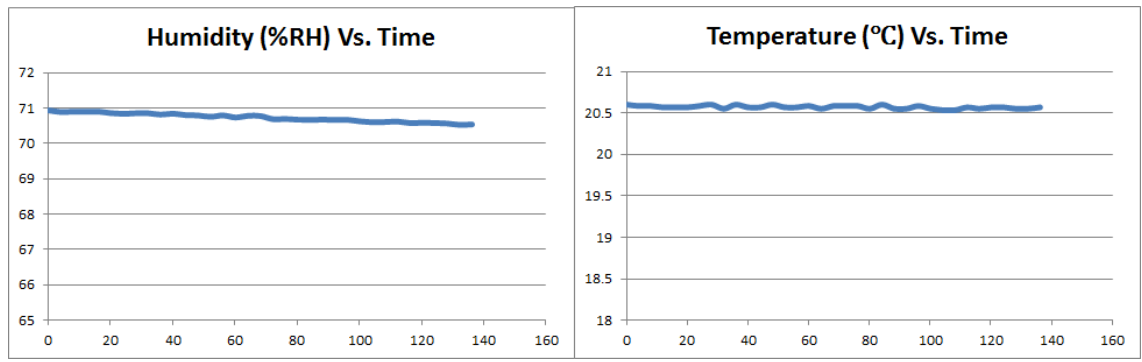

Figure 12: Temperature and humidity results for indoor measurements. 
Thus, the functionality of the system was verified for both locations. These results form a base of the designed weather station and monitoring system, where the main environmental parameters have already been integrated and tested, with an Air Quality Index that compares the measurand levels with the local standards and display warnings in case of exceeding the pre-set safety thresholds. In other words, this system can be expanded to include the gas sensors once they are calibrated to monitor the effects of such gases on health and take autonomous decisions based on the results.

Figure 13 depicts the solar irradiance and ambient temperature output.
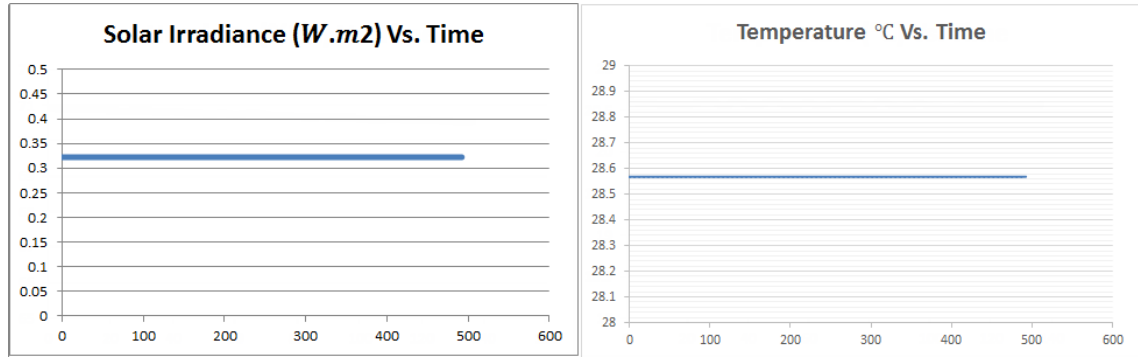

Figure 13: Solar irradiance and temperature output (outdoor).

In general, the accuracy of the system with its current shape is moderate. This accuracy can be further improved by applying more complex modeling methods in LabVIEW such as for the dust sensor. Moreover, the quality of some sensors (e.g. humidity) is not the best. Thus, it is recommended to select more sensitive and accurate sensor for the final version of the system.

\section{Conclusion and further work}

In conclusion, a comprehensive wireless system for monitoring and measuring system was designed and built. This system deals with parameters that affect our lives, environment and industries. The complete system, software \& hardware has been successfully implemented and tested in a pilot study in a selected site within Qatar University Campus. The developed system functioned properly. All environmental and climatic were monitored wirelessly indoor and outdoor. Nonetheless, one important issue that is needs further study is regarding the gas sensors calibration; providing and mixing the gasses. Implementing the system in different locations, minimizing the energy consumption and acquiring the data for long period are of the main steps forward in this project.

\section{References}

[1] Buttrich. S. "Introduction to Wireless Sensor Networks", University of Copenhagen, 2011.

[2] Bener et al., The impact of air pollution on hospital admission for respiratory and cardiovascular diseases in an oil and gas-rich country, Eur. Ann. Allergy Clin. Immunol., Vol. 41, pp. 80-84, 2009. 
[3] F. S. Al-Anzi and A. A. Salman, Correlation of Asthma Symptoms with Prevalence of Indoor $\mathrm{NO}_{2}$ Concentration in Kuwait, Journal of Environmental Protection, Vol. 2, pp. 186-193, 2011.

[4] Lian-Yu Lin et al., The effects of indoor particle exposure on blood pressure and heart rate among young adults: An air filtration-based intervention study, Atmospheric Environment, Vol. 45, pp. 5540-44, 2011.

[5] Qatar National Development Strategy 20112016 (Towards Qatar National Vision 2030), Gulf Publishing and Printing Company, 2011.

[6] http://www.qatarisbooming.com/2011/04/01/qatar-to-develop-policy-tomonitor-air-quality/

[7] Reiche. D. "Energy policies of Gluf Cooperation Council (GCC) countries", Wuppertal Institute, 2010.

[8] Touati. F, Al-Hitmi. M, Bouchech. H. "Stud of the effects of dust, relative humidity, and temperature on solar PV performance in Doha", International Journal of Green Energy Vol. 10, Issue 5, 2013.

[9] WHO guidelines for indoor air quality: selected pollutants, World Health Organization, 2010 (http://www.euro.who.int/_data/assets/pdf_file/0009 /128169/e94535.pdf).

\section{Acknowledgment}

This publication was made possible by UREP grant \# 14 - 034 - 2-011 from the Qatar National Research Fund (a member of Qatar Foundation). The statements made herein are solely the responsibility of the authors. 